OPEN

SUBJECT AREAS:

MEMBRANE STRUCTURE

AND ASSEMBLY

PHOTOSYSTEM II

Received

22 November 2013

Accepted

3 February 2014

Published

24 February 2014

Correspondence and requests for materials should be addressed to W.S.C. (Fred.Chow@ anu.edu.au)

* These authors contributed equally to this work.

\section{Entropy and biological systems: Experimentally-investigated entropy-driven stacking of plant photosynthetic membranes}

\author{
Husen Jia*, John R. Liggins* \& Wah Soon Chow
}

Division of Plant Science, R. N. Robertson Building (46), Research School of Biology, The Australian National University, Canberra, ACT 0200, Australia.

According to the Second Law of Thermodynamics, an overall increase of entropy contributes to the driving force for any physicochemical process, but entropy has seldom been investigated in biological systems. Here, for the first time, we apply Isothermal Titration Calorimetry (ITC) to investigate the $\mathrm{Mg}^{2+}$-induced spontaneous stacking of photosynthetic membranes isolated from spinach leaves. After subtracting a large endothermic interaction of $\mathrm{MgCl}_{2}$ with membranes, unrelated to stacking, we demonstrate that the enthalpy change (heat change at constant pressure) is zero or marginally positive or negative. This first direct experimental evidence strongly suggests that an entropy increase significantly drives membrane stacking in this ordered biological structure. Possible mechanisms for the entropy increase include: (i) the attraction between discrete oppositely-charged areas, releasing counterions; (ii) the release of loosely-bound water molecules from the inter-membrane gap; (iii) the increased orientational freedom of previously-aligned water dipoles; and (iv) the lateral rearrangement of membrane components.

tacking of photosynthetic membranes (thylakoids) to form grana interconnected by non-stacked thylakoids, bathed in an aqueous stroma, is ubiquitous in plants, being required for fine-tuning (a) photosynthesis, (b) photoprotection and (c) acclimation to environments ${ }^{1-4}$. Advantages of granal stacking include: (1) an extremely large membrane surface-to-volume ratio to accommodate a high density of chlorophyll (Chl) to promote light-harvesting ${ }^{5}$; $(2)$ a spatial separation of the two photosystems ${ }^{6}$ which limits excessive spillover of excitation energy from Photosystem II (PS II, responsible for oxygen evolution) to PS $\mathrm{I}^{1,2,7}$; (3) enhancement of non-cyclic photophosphorylation to increase the "energy currency" ; (4) regulation of non-photochemical dissipation for protection against excess light ${ }^{9}$; (5) delay of premature degradation of D1 protein in the PS II reaction centre at sustained high irradiance ${ }^{10} ;(6)$ regulation of linear versus cyclic electron transport ${ }^{11}$; and (7) a potential increase in photosynthetic capacity for a given chloroplast composition ${ }^{4}$.

Here we monitored $\mathrm{Mg}^{2+}$-induced stacking of spinach photosynthetic membranes to address the question of what thermodynamic attractive force drives granal stacking. Granal stacking should depend on the net sum of (1) hydration repulsion, (2) electrostatic repulsion, (3) van der Waals attraction, and (4) entropy-driven attraction. Hydration repulsion is very short-ranged, originating from the displacement of structured water molecules around charged groups on membranes surfaces ${ }^{12}$. It partly determines the separation between stacked thylakoids, recently estimated as $3.2 \mathrm{~nm}^{13}$.

Electrostatic repulsion, caused by the net negative charge on the outer surface of thylakoid membranes, is a major factor opposing granal stacking ${ }^{5,14-18}$. A net negatively-charged membrane in an electrolyte is surrounded by a diffuse layer of counterions and is therefore electrically neutral; however, bringing two such membranes together would squeeze the diffuse counterion layers which then resist with an osmotic pressure. This is the origin of the electrostatic repulsion between two membrane surfaces in an aqueous ionic medium, as distinct from air. Notably, the electrostatic repulsion can be decreased by adding ionic solutes to provide charge screening s.15-20. $^{4}$

However, since even in the presence of cations the electrostatic force is still repulsive, an attractive force that exceeds the electrostatic repulsion is required for granal stacking. van der Waals attraction is long-range, often dominated by the London dispersion force among instantaneously-induced dipoles. There has been only one published attempt at calculating it for thylakoids ${ }^{18,21}$. Significantly, the calculated van der Waals attraction is vastly 


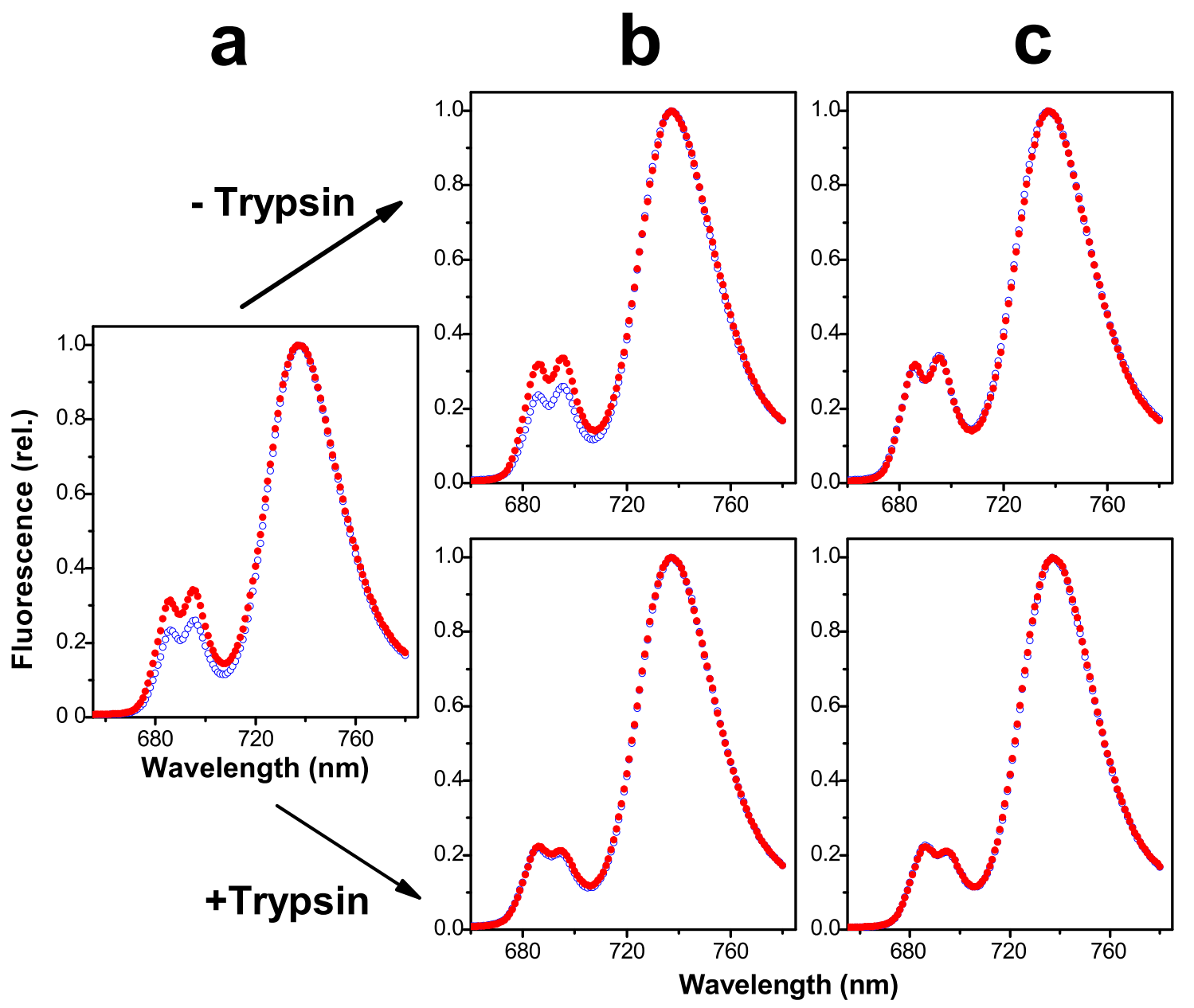

Figure 1 Fluorescence emission spectra at $77 \mathrm{~K}$ of previously-unstacked membranes placed in the absence (blue open circles) or presence (red closed circles) of $5 \mathrm{mM} \mathrm{MgCl}_{2}$. Samples were taken (a) from the thylakoid stock suspension before addition to the ITC sample cell; (b) from the ITC sample cell immediately after addition to the cell but before the commencement of calorimetric titration; and (c) from the ITC sample cell at the end of titration. The excitation wavelength was $440 \mathrm{~nm}$. Spectra were obtained as described in Methods.

weaker than the electrostatic repulsion; therefore, the van der Waals attraction might not effect membrane stacking. What then is the main attractive force?

Chow ${ }^{14}$ hypothesized that a high concentration of stromal macromolecules, such as the abundant ribulose 1,5-bisphosphate carboxylase/oxygenase (Rubisco) complex or ribosomes, results in an entropically-driven force (depletion attraction): granal stacking allows greater volume for free diffusion of stromal macromolecules in a crowded environment. This was observed in thylakoids in vitro, using negatively-charged bovine serum albumin or neutral dextran as the macromolecules ${ }^{15}$. Nevertheless, the question remains as to what attractive force overcomes the electrostatic repulsion when $\mathrm{MgCl}_{2}$, rather than macromolecules, is added to unstacked thylakoids.

To investigate the nature of the attractive force driving $\mathrm{Mg}^{2+}$. induced granal stacking, we used the highly sensitive technique of ITC to directly measure the enthalpy change $\left(\Delta H_{\text {exp }}\right)$. We also obtained the theoretical maximum and minimum sum of the component enthalpy changes ( $\Delta H_{\text {tot }}$ max and $\Delta H_{\text {tot }}$ min) from published component forces between thylakoid membranes as functions of inter-membrane separation. The results show that the theoretical enthalpy change is slightly positive and that the experimental enthalpy change, while the mean value obtained from several experiments is slightly negative, could either be zero, slightly positive or slightly negative within experimental error. This is the first direct experimental evidence which strongly suggests that an entropy increase might indeed drive the spontaneous, $\mathrm{Mg}^{2+}$-induced stacking of membranes in this intriguingly ordered biological structure. Possible mechanisms for the entropy increase are discussed.

\section{Results}

Monitoring the stacking of thylakoids in isolated envelope-free chloroplasts. Chl fluorescence emission spectra of a dilute suspension of envelope-free chloroplasts at $77 \mathrm{~K}$ exhibit peaks: a double peak emitted by PS II at 686 and $696 \mathrm{~nm}$, and a single peak emitted by PS I at $736 \mathrm{~nm}$ (Figure 1). The double peak is lowered relative to the single peak upon unstacking of the thylakoids prior to freezing at $77 \mathrm{~K}$. This is because unstacking induces a random mixing of the two photosystems within the membrane system, and increases "spillover" of excitation energy from PS II to PS I, thereby lowering the Chl fluorescence emission by PS II. Therefore, the height of the double peak relative to that of the single peak is a convenient measure of the extent of granal stacking, a higher double peak indicating a greater extent of granal stacking. Figure 1 shows the lower double peak exhibited by unstacked membranes (blue open circles). Before and during the ITC experiments, small aliquots of the membrane suspensions were taken from three sampling times. Figure 1 shows that in the presence of $\mathrm{MgCl}_{2}$ (red closed circles) at any sampling time, the membranes were stacked (high double peak) in the absence but not the presence of a low molar ratio of trypsin to Chl. 
Table 1 | Experimental $\Delta H_{\text {exp }}$ and theoretical values of the component enthalpy changes of $\mathrm{Mg}^{2+}$-induced stacking of thylakoid membranes. Values of the enthalpy changes are in $\mathrm{k} \mathrm{mol}^{-1} \mathrm{Chl}$. Endothermic changes are positive, and exothermic changes negative. Column $1\left(\Delta H_{\text {exp }}\right)$ is the experimental enthalpy change of thylakoid stacking, calculated as described in Methods, averaged from a total of six days' experiments, displayed as mean \pm S.D. Columns A-D refer to $\Delta H$ after integration of the electrostatic repulsion calculated at constant surface charge density $(\sigma)$ or constant surface potential $(\psi)$. The values in the rows marked $2 \mathrm{~nm}, 3 \mathrm{~nm}$ and $4 \mathrm{~nm}$ were obtained by integrating the curves from membrane separations (Gap) of $2 \mathrm{~nm}, 3 \mathrm{~nm}$ and $4 \mathrm{~nm}$, respectively, to the maximum distance in the calculations ${ }^{21}$; the values shown in square brackets were obtained by extrapolation to separations below those in the calculations ${ }^{21}$ (see Methods). The row marked $3 \mathrm{~nm}$ has been shaded because the most recent estimate yielded $3.2 \mathrm{~nm}$ for the inter-membrane gap ${ }^{13}$. $\Delta H_{\text {tot }}$ is the algebraic sum of the electrostatic, van der Waals and hydration enthalpy changes: $\Delta H_{\text {tot }}(\max )=\Delta H_{\sigma}(1)+\Delta H_{\text {hyd }}+\Delta H_{\mathrm{vdW}}(2)$. $\Delta H_{\text {tot }}(\min )=\Delta H_{\psi}(2)+\Delta H_{\text {hyd }}+\Delta H_{\mathrm{vdW}}(1) . \mathrm{V}_{\mathrm{pr}}$ is the volume fraction of protein in the membrane, and $\varepsilon$ is the assumed dielectric constant

\begin{tabular}{|c|c|c|c|c|c|c|c|c|c|c|}
\hline \multirow{3}{*}{$\Delta H_{\exp }$} & \multirow{3}{*}{ Gap } & (A) & (B) & (C) & (D) & (E) & (F) & (G) & \multirow{3}{*}{$\frac{\Delta H_{\text {tot }}}{\text { (max) }}$} & \multirow[b]{2}{*}{$\Delta H_{\mathrm{to}}$} \\
\hline & & $\Delta H_{\sigma}$ & $\Delta H_{\psi}$ & $\Delta H_{\sigma}$ & $\Delta H_{\psi}$ & \multirow{2}{*}{$\Delta H_{\text {hyd }}$} & $\Delta H_{\mathrm{vdw}}$ & $\Delta H_{\mathrm{vdW}}$ & & \\
\hline & & (1) & (1) & (2) & (2) & & (1) & (2) & & (min) \\
\hline \multirow{4}{*}{$\begin{array}{l}-0.20 \\
\pm 0.51\end{array}$} & & $\begin{array}{c}1 \mathrm{mM} \\
\mathrm{K}^{+} \\
50 \mu \mathrm{M} \\
\mathrm{Mg}^{2+}\end{array}$ & $\begin{array}{c}1 \mathrm{mM} \\
\mathrm{K}^{+} \\
50 \mu \mathrm{M} \\
\mathrm{Mg}^{2+}\end{array}$ & $\begin{array}{c}10 \mathrm{mM} \\
\mathrm{K}^{+} \\
5 \mathrm{mM} \\
\mathrm{Mg}^{2+}\end{array}$ & $\begin{array}{c}10 \mathrm{mM} \\
\mathrm{K}^{+} \\
5 \mathrm{mM} \\
\mathrm{Mg}^{2+}\end{array}$ & $\begin{array}{c}10 \mathrm{mM} \\
\mathrm{K}^{+} \\
5 \mathrm{mM} \\
\mathrm{Mg}^{2+}\end{array}$ & $\begin{array}{l}\varepsilon=3 \\
V_{\mathrm{pr}} \\
=0.6\end{array}$ & $\begin{array}{c}\varepsilon=2.5 \\
V_{p r} \\
=0.4\end{array}$ & & \\
\hline & $2 \mathrm{~nm}$ & {$[1.37]$} & {$[0.68]$} & {$[1.21]$} & {$[0.66]$} & 0.015 & -0.037 & -0.021 & 1.36 & 0.64 \\
\hline & $3 \mathrm{~nm}$ & {$[0.68]$} & {$[0.45]$} & {$[0.49]$} & {$[0.35]$} & 0 & -0.013 & -0.006 & 0.67 & 0.34 \\
\hline & $4 \mathrm{~nm}$ & 0.36 & 0.29 & 0.22 & 0.19 & 0 & -0.007 & -0.002 & 0.36 & 0.18 \\
\hline
\end{tabular}

Experimental enthalpy change $\Delta H_{\exp }$ associated with granal stacking. $\mathrm{MgCl}_{2}$ was titrated into suspensions of unstacked thylakoids, some of which had had minute concentrations of trypsin (1:3,000 molar ratio of trypsin to chlorophyll) added to them (trypsin is known to render thylakoid membranes incapable of stacking ${ }^{22,23}$ ), at a membrane concentration equivalent to $0.2 \mathrm{mM}$ Chl (see Methods). Subsequent Chl fluorescence analysis of samples frozen at $77 \mathrm{~K}$ revealed that the membrane suspensions without trypsin had stacked during the course of the ITC experiment while those with added trypsin had not. The positive enthalpy signal of the raw ITC plot (see Methods) reveals that the overall interaction of $\mathrm{MgCl}_{2}$ with the thylakoid membranes was endothermic. The enthalpy change of the interaction was about $3.4 \mathrm{~kJ}$ per mol $\mathrm{Chl}$ at a membrane concentration equivalent to $0.2 \mathrm{mM}$ Chl, decreasing markedly to $1.25 \mathrm{~kJ} \mathrm{~mol}^{-1} \mathrm{Chl}$ at $1 \mathrm{mM}$ $\mathrm{Chl}$, and $0.33 \mathrm{~kJ} \mathrm{~mol}^{-1} \mathrm{Chl}$ at $3.3 \mathrm{mM} \mathrm{Chl}$ (data not tabulated). At $1 \mathrm{mM} \mathrm{Chl}$, the interaction was $50 \%$ complete at about $1 \mathrm{mM} \mathrm{MgCl}_{2}$ (data not shown). Notably, virtually identical plots were obtained regardless of whether the membranes stacked or did not stack (see Methods). This shows that the endothermic interaction is predominantly unrelated to stacking but is presumably due to the interaction of $\mathrm{Mg}^{2+}$ ions (or $\mathrm{Cl}^{-}$ions) with membrane components.

We determined the enthalpy change of membrane stacking (at $0.2 \mathrm{mM} \mathrm{Chl}$ ) by subtracting the $\Delta \mathrm{H}$ values obtained from the ITC plots for those membranes which were shown by subsequent $\mathrm{Chl}$ fluorescence spectral analysis not to have stacked during the course of the experiment from those obtained from the ITC plots for those membranes which were shown to have stacked. This yielded an average enthalpy change of $\Delta H_{\exp }=-0.20 \pm 0.51 \mathrm{~kJ} \mathrm{~mol}^{-1} \mathrm{Chl}$ (first column of Table 1).

Thus, the magnitude of $\Delta H_{\exp }$ was very small: while the mean value in the first column of Table 1 was marginally below zero, the actual value could either be zero or marginally above or below zero within experimental error. Since the Gibbs free energy change $(\Delta G)$ for the spontaneous stacking of thylakoids upon adding $\mathrm{Mg}^{2+}$ at an absolute temperature $T$ must be appreciably negative, this observation implies that an increase in entropy $(\Delta S)$ probably makes a significant contribution to the negative Gibbs free energy change $\Delta G=\Delta H-T \Delta S$.
Theoretical sum of component enthalpy changes $\Delta H_{\text {tot }}$ associated with granal stacking. The theoretical calculations of the electrostatic repulsion made by Rubin et al. $(1981)^{21}$ and the hydration repulsion and the van der Waals attraction between thylakoid membranes calculated by Sculley et al. $(1980)^{18}$ have been reproduced in a linear plot in Figure 2 as functions of the distance between

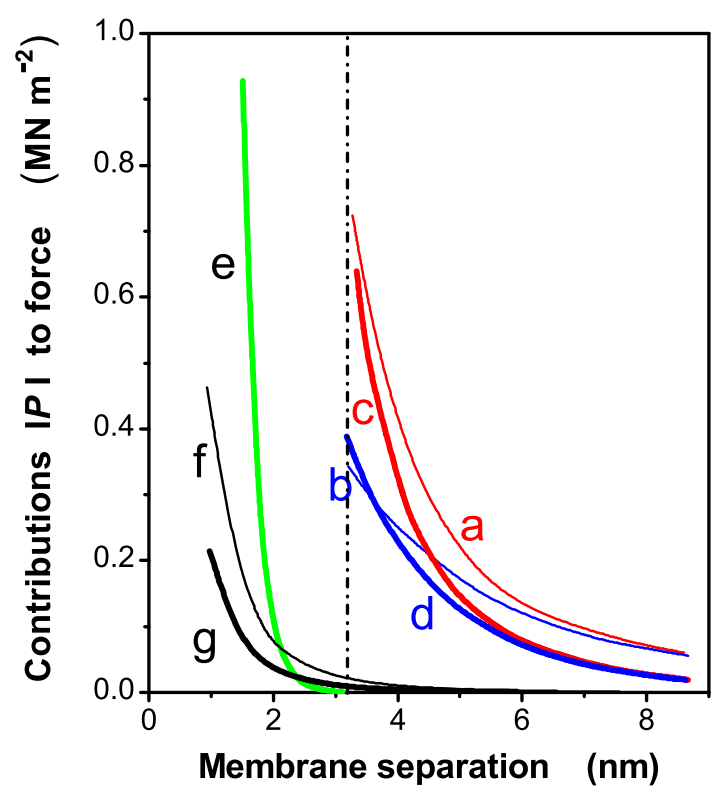

Figure $2 \mid$ Theoretical calculations of the contributions, $|P|$, to the force between two membranes as a function of separation. Re-plotted on a linear scale from Figure 8 of Rubin et al. ${ }^{21}$. The curves $a, b, c$ and $d$ represent the electrostatic repulsion in two ionic media (one stacking and the other unstacking), calculated at either constant surface charge density or constant surface potential. Curve e denotes the hydration repulsion. Curves $\mathrm{f}$ and $\mathrm{g}$ denote the van der Waals attraction, corresponding to dielectric constants of 3 and 2.5, respectively, with volume fractions of protein (Vpr) in the membrane of 0.6 and 0.4 , respectively. The dashed vertical line denotes a very recent estimation of membrane separation ${ }^{13}$. 
membranes; the linear plot gives a better visual impression than a semi-log plot $^{18,21}$ of the magnitudes of these component forces. The various electrostatic forces (curves a, b, c and d) and the hydration force (curve e) are repulsive while the two van der Waals curves ( $f$ and g) are attractive. The theoretical enthalpy changes of stacking produced by these forces were calculated by integrating the curves in Figure 2 to three membrane-separations (gap). The theoretical $\Delta H_{\text {tot }}$ consists of endothermic $\Delta H$ components associated with electrostatic repulsion and hydration repulsion, and an exothermic component associated with van der Waals attraction. Table 1 depicts the theoretical values of the total enthalpy $\left(\Delta H_{\text {tot }}\right)$ for membrane stacking calculated for three inter-membrane gaps. For a gap of $3 \mathrm{~nm}$ (shaded row in Table 1$)$, the total enthalpy change $\left(\Delta H_{\text {tot }}\right)$ for membrane stacking ranged from a lower limit, $\Delta H_{\text {tot }}(\mathrm{min})$, of $0.34 \mathrm{~kJ} \mathrm{~mol}^{-1} \mathrm{Chl}$ to an upper limit, $\Delta H_{\text {tot }}(\max )$, of $0.67 \mathrm{~kJ} \mathrm{~mol}^{-1}$ Chl. For a gap of $4 \mathrm{~nm}$ (Table 1), the lower limit was $0.18 \mathrm{~kJ} \mathrm{~mol}^{-1}$ $\mathrm{Chl}$ while the upper limit was $0.36 \mathrm{~kJ} \mathrm{~mol}^{-1} \mathrm{Chl}$. The experimentally determined value, $-0.20 \pm 0.51 \mathrm{~kJ} \mathrm{~mol}^{-1} \mathrm{Chl}$, falls just outside the range for a $3 \mathrm{~nm}$ gap but within the range for a $4 \mathrm{~nm}$ gap, which is consistent with the recently estimated gap distance of $3.2 \mathrm{~nm}^{13}$. Further, if the dielectric constant $\varepsilon$ of the thylakoid membrane were greater than $3, \Delta H_{\mathrm{vdW}}$ (Table 1) would be more negative, making the $\Delta H_{\text {tot }}$ less positive and the experimental value more within the range of theoretical estimates. Hence, the positive theoretical $\left(\Delta H_{\text {tot }}\right)$ enthalpy change and the near zero experimental $\left(\Delta H_{\exp }\right)$ enthalpy change strongly imply that a significant entropic driving force is required to achieve an appreciably negative $\Delta G$ and to effect spontaneous stacking on adding $\mathrm{MgCl}_{2}$.

\section{Discussion}

How could an entropy-driven attraction arise on adding $\mathrm{Mg}^{2+}$ to unstacked isolated thylakoids? Here, we suggest four ways whereby entropy can be increased.

Firstly, the light-harvesting complex II (LHC-II) is a major component of higher plant thylakoid membranes. The outer stromafacing surface of an LHC-II trimer consists of discrete areas of net positive charge separated from areas of net negative charge ${ }^{24}$. A positive area on one LHC-II trimer could interact non-specifically with a negative area on an LHC-II trimer in the opposite membrane; this "Velcro effect" could play a major role in granal stacking ${ }^{24}$. A similar idea ${ }^{22}$ was advanced earlier, though its "contact mechanism" requires interaction between specific groups of opposite charge. Each positively-charged or negatively-charged area is surrounded by a diffuse aqueous layer containing counterions. When two membranes experience less electrostatic repulsion upon adding screening cations such as $\mathrm{Mg}^{2+}$, and begin to approach each other such that areas of opposite charge face each other (Figure 3a), counterion pairs are released from the inter-membrane gap to diffuse freely as individual ions in the bulk medium, thereby gaining entropy ${ }^{25}$.

Secondly, upon adding $\mathrm{MgCl}_{2}$, as two domains of opposite charge come closer, loosely-bound water molecules could be released from the inter-membrane gap to the bulk medium (Figure $3 \mathrm{~b}$ ), thus increasing entropy. Such release occurs because the end of a water dipole pointing away from one domain is attracted by an approaching domain of opposite charge.

Thirdly, in an unstacking medium (without $\mathrm{Mg}^{2+}$ ), the local electric field strength $(E)$ near a negatively-charged (or a positivelycharged) area may be high enough to align water dipoles locally, thereby restricting their orientational freedom (Figure 3c). Assuming that the local surface charge density of a negativelycharged area is 10 times the overall net negative surface charge density $(\sigma)$ of the stroma-facing membrane surface, the estimated magnitude of $E$ near the charged area in an unstacking electrolyte can be calculated. Figure 4 depicts the variation of $E$ with distance from a negatively-charged area of LHC-II for the two ionic compositions specified in the figure legend. In the unstacking $\mathrm{Z}^{+} / \mathrm{Z}^{-}$electrolyte, the
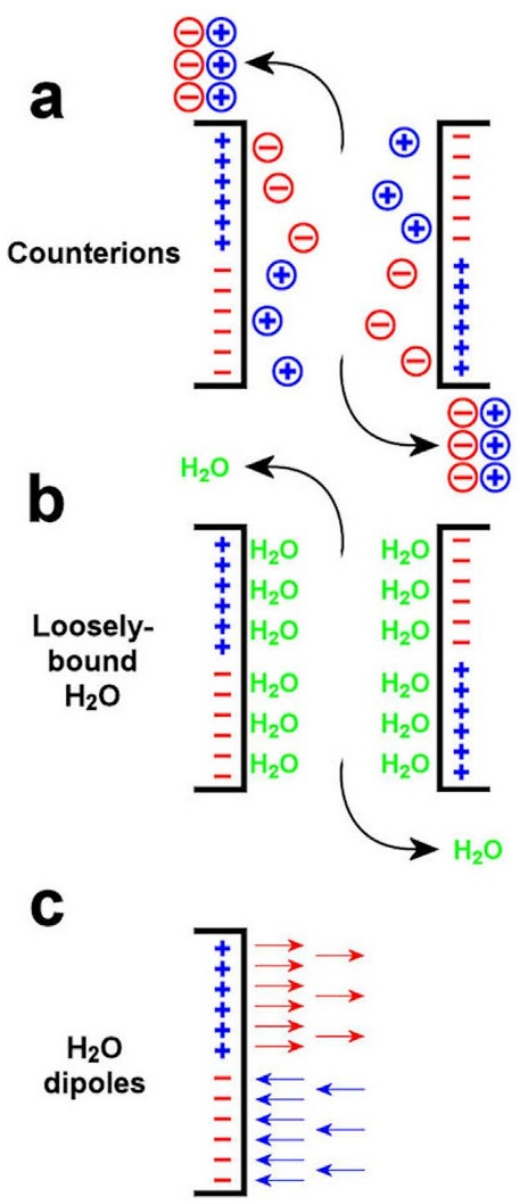

Figure $3 \mid$ Mechanisms by which entropy can be increased by the stacking of two thylakoid membranes. (a), areas of opposite charge approach each other in a Velcro effect, expelling pairs of counterions which then diffuse freely as individual ions. (b), loosely-bound water molecules are released from the inter-membrane gap when areas of opposite charge approach each other, thereby diffusing freely. (c), in an unstacking ionic medium, the electric field strength adjacent to the membrane surface is sufficient to align water dipoles, but is diminished on adding $\mathrm{Mg}^{2+}$ (details in Methods and Discussion); loss of alignment increases the orientational freedom of water dipoles.

local electric field strength $E$ at the negatively-charged area surface is approximately $1.14 \mathrm{GV} \mathrm{m}^{-1}$ (upper curve). A water molecule with a dipole moment of $6.17 \times 10^{-30} \mathrm{C} \mathrm{m}$ will have minimum potential energy $1.14 \mathrm{GV} \times 6.17 \times 10^{-30} \mathrm{C} \mathrm{m}=7.0 \times 10^{-21} \mathrm{~J}$, with a magnitude almost twice the kinetic energy per water molecule of $k T=4.1$ $\times 10^{-21} \mathrm{~J}$, where $k$ is Boltzmann's constant. Therefore, the electric field strength near a negatively-charged area on the stroma-facing surface of LHC-II could be high enough to align water dipoles against their random orientational motion. Upon adding $\mathrm{Mg}^{2+}$, however, $E$ is reduced by a factor of about 4 (lower curve), and the alignment is almost certainly lost. Similarly, a positively-charged area of the stroma-facing surface of LHC-II could induce an electric field strong enough to align water molecules in an unstacking buffer, this time in the opposite orientation; $E$ could again be much diminished in a stacking medium. In either case, the gain in orientational freedom of water dipoles upon adding $\mathrm{MgCl}_{2}$ could then contribute to an increase in entropy that drives the stacking of thylakoids. The increased orientational freedom of water molecules on adding $\mathrm{MgCl}_{2}$ should compensate for the rise in potential energy of water dipoles (reaching a less negative value) in a diminished electric field; in turn, the rise in potential energy of water dipoles should promote 


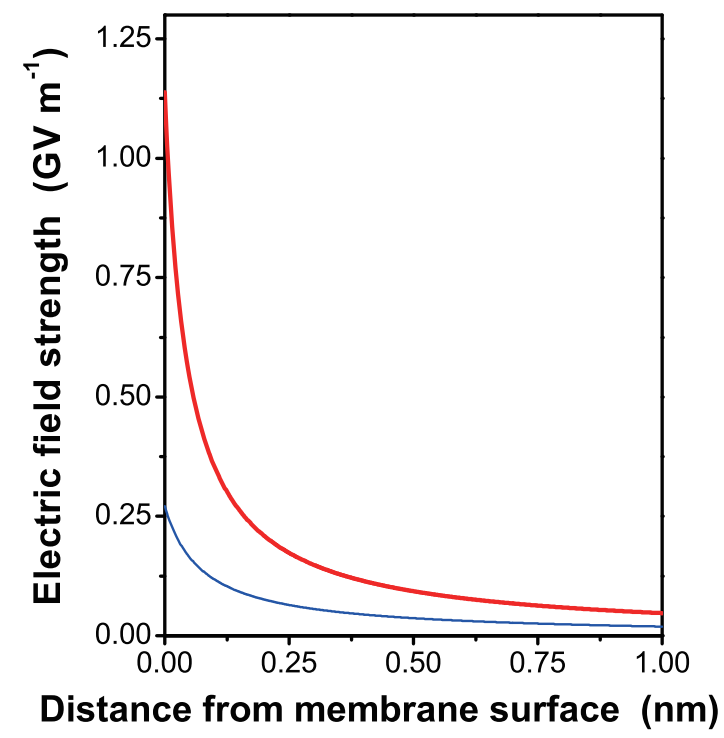

Figure $4 \mid$ The electric field strength as a function of distance from a charged surface with a local surface charge density of $-0.25 \mathrm{C} \mathrm{m}^{-2}$, calculated in Methods. This value is assumed to be ten times the net negative overall surface charge density on thylakoid membranes. The upper thick curve refers to an unstacking ionic medium $(20 \mathrm{mM}$ monovalent cation, $20 \mathrm{mM}$ monovalent anion), and the lower thin curve to a stacking medium (20 mM monovalent cation, $30 \mathrm{mM}$ monovalent anion and $5 \mathrm{mM}$ divalent cation).

the above-mentioned release of loosely-constrained water molecules into the bulk medium, thereby increasing entropy.

Fourthly, there is the entropy-driven attraction (depletion attraction $^{26}$ ) by which large membrane-spanning complexes ('large particles'), such as PS II and LHC-II, are pushed together to form dimers and trimers, respectively, within a single membrane, so as to free up volume for diffusion of smaller intra-membrane 'small particles' (lipids and plastoquinone) ${ }^{14}$. The aggregation of PS II and LHC-II will position the protein complexes on opposite membranes for the Velcro effect. Additionally, there is more entropy to gain from the aggregation of PS II and LHC-II if the acyl chains of the lipids are highly unsaturated compared with saturated acyl chains: the bend in unsaturated acyl chains due to cis-double bonds contributes more to entropy if the unsaturated acyl chains are given more space in which to flex freely, when compared with saturated acyl chains. Consistent with this expectation, granal stacking is enhanced in thylakoids with highly-unsaturated lipids ${ }^{27}$.

Finally, it is intriguing how, in previously-unstacked thyakoids, stacking may be initiated by adding $\mathrm{Mg}^{2+}$. The membranes have to be brought to a sufficiently small separation for the Velcro effect to operate. We envisage that an unstacked membrane system "wobbles", such that occasionally a localized protein-rich domain on one membrane comes sufficiently close to one on the opposite membrane. The gain in entropy from a localized Velcro effect would lower the free Gibbs energy, enabling the adhesion to propagate to other regions of the membrane system.

$\mathrm{Mg}^{2+}$ and $\mathrm{K}^{+}$being abundant in the chloroplast stroma ${ }^{28}$, the in vitro effect of $\mathrm{Mg}^{2+}$ on inducing thylakoid stacking observed here, and of high concentrations of monovalent cations reported elsewhere ${ }^{21,29}$, should also apply in vivo. Our study of what strongly appears to be an entropy-driven attraction in $\mathrm{Mg}^{2+}$-induced granal stacking, together with entropy-driven depletion attraction in the presence of macromolecules ${ }^{15}$, highlights the importance of the unifying concept of an entropy-driven attraction that determines chloroplast ultrastructure generally, in vivo and in vitro, thereby regulating chloroplast function indirectly. This and other hitherto rare investigations of the contributions of entropy, as formulated by the Second law of Thermodynamics, to the driving forces of biological processes will lead to a deeper understanding of biological systems.

\section{Methods}

Plant growth. Spinacea oleracea L. (cv. Yates hybrid 102) plants were grown in a glasshouse during spring at approximately $28 / 15^{\circ} \mathrm{C}$ (day/night) under about $10 \%$ natural light to minimize starch content.

Preparation of thylakoid membranes. Leaves were homogenized at $6^{\circ} \mathrm{C}$ in a buffer containing $20 \mathrm{mM}$ Tricine- $\mathrm{KOH}$ (pH 8.4), $0.3 \mathrm{M}$ sorbitol, $10 \mathrm{mM}$ EDTA, $10 \mathrm{mM}$ $\mathrm{NaHCO}_{3}, 5 \mathrm{mM} \mathrm{MgCl}, 10 \mathrm{mM} \mathrm{KCl}, 4.5 \mathrm{mM}$ sodium ascorbate and $0.5 \%$ bovine serum albumin. The homogenate was filtered through 6 layers of muslin and centrifuged at $3,000 \times g$ for $2 \mathrm{~min}$ at $4^{\circ} \mathrm{C}$. After re-suspension in $20 \mathrm{mM}$ Tricine$\mathrm{KOH}$ (pH 7.6), 0.3 M sorbitol, $5 \mathrm{mM} \mathrm{MgCl}_{2}$ and $2.5 \mathrm{mM}$ EDTA, the chloroplasts were osmotically shocked in $5 \mathrm{mM} \mathrm{MgCl}_{2}$ for $1 \mathrm{~min}$. An equal volume of medium was added to give $50 \mathrm{mM}$ Hepes- $\mathrm{KOH}$ (pH 7.6), $0.3 \mathrm{M}$ sorbitol, $2.5 \mathrm{mM} \mathrm{MgCl}_{2}$ and $10 \mathrm{mM} \mathrm{KCl}$. Following centrifugation at $3,000 \times g$ for $2 \mathrm{~min}$ at $4^{\circ} \mathrm{C}$, the thylakoid membranes were re-suspended in a small volume of buffer containing $20 \mathrm{mM}$ Tricine-KOH (pH 7.6), $2.5 \mathrm{mM}$ EDTA and $0.3 \mathrm{M}$ sorbitol.

Chlorophyll determination. Chlorophyll was determined in buffered $80 \%$ acetone according to Porra et al. ${ }^{30}$.

Monitoring stacking of the thylakoid membranes by Chl fluorescence spectral analysis. Small aliquots of the membrane suspensions were taken: (a) from the thylakoid stock suspension before addition to the ITC sample cell; (b) from the ITC sample cell immediately after addition to the cell but before the commencement of calorimetric titration; and (c) from the ITC sample cell at the end of titration (Figure 1). Each aliquot was divided into two portions. To one portion was added $\mathrm{MgCl}_{2}$ (final concentration $5 \mathrm{mM}$, Figure 1, red closed circles) which would induce stacking of the membranes if they were capable of stacking. The other portion had no $\mathrm{MgCl}_{2}$ added (blue open circles). Each portion was diluted to $2 \mu \mathrm{M} \mathrm{Chl}$ in the ITC buffer containing $20 \mathrm{mM}$ Hepes ( $\mathrm{pH} 7.6), 0.3 \mathrm{M}$ sorbitol and $10 \mathrm{mM} \mathrm{KCl}$, with or without $5 \mathrm{mM} \mathrm{MgCl}_{2}$, and frozen for $77 \mathrm{~K}$ fluorescence spectral analysis to determine the extent of membrane stacking.

A spectrofluorimeter (SLM-8100, Spectronic, Rochester, NY) excited the membrane samples at $440 \mathrm{~nm}$, to yield the PS II double emission peak (686 nm and $696 \mathrm{~nm})$ and the PS I peak (736 nm) at $77 \mathrm{~K}$, corrected for wavelength-dependent instrument response. The ratio PS II peak/PS I peak represents the partitioning of absorbed light energy between the two photosystems: the greater the extent of stacking, the higher the PS II double peak relative to the PS I peak.

Isothermal titration calorimetry (ITC). ITC experiments were conducted using a Microcal VP-ITC (MicroCal, Northampton, USA). The stock membrane suspension was diluted to $0.2 \mathrm{mM} \mathrm{Chl}$, unless otherwise specified, in the ITC buffer. The membrane suspension was degased, loaded into the ITC sample cell (volume $1.44 \mathrm{~mL}$ ), and kept in darkness. Aliquots of a $50 \mu \mathrm{M}$ trypsin solution were added to some samples (molar ratio of 1 trypsin: 3,000 Chl). Incubation with trypsin was for $\sim 30$ minutes before the addition of $\mathrm{MgCl}_{2}(25 \mathrm{mM})$ from a syringe (capacity $300 \mu \mathrm{L}$ ). Each day, at least three experiments were performed without added trypsin and at least two were performed with added trypsin. The experiment was conducted at $25^{\circ} \mathrm{C}$ with the syringe needle stirring at $260 \mathrm{rpm}$, and consisted of 3 injections, each of $95 \mu \mathrm{L}$, with a duration of $190 \mathrm{~s}$, spaced 10 minutes apart, unless otherwise specified (Fig. 5a).

Calculation of the experimental enthalpy change of membrane stacking from the ITC data. The pre-injection and post-injection baselines were extrapolated across each injection (Figure 5a, b, d and e). Linear fits were made to the segments of the plot immediately before, and at the end of, each injection (Figure $5 \mathrm{~b}$ and e) and the slopes measured. A cubic polynomial continuous with the pre-injection and the postinjection segments was calculated, the instantaneous slopes of the polynomial equalling the slopes of the pre-injection and the post-injection segments. The extrapolated baseline was then subtracted from the raw heat capacity plot to give the heat capacities $\left(\mu \mathrm{J} \mathrm{s}^{-1}\right)$ (Figure $5 \mathrm{c}$ and $\mathrm{f}$ ). The enthalpy change of each injection was obtained by integration. The interaction of $\mathrm{Mg}^{2+}$ ions with the thylakoid membrane was deemed complete by the end of the second injection; therefore, the raw enthalpy of the third injection was taken to represent the heat of dilution of the third injection of $\mathrm{MgCl}_{2}$ solution. This value, corrected for the progressive dilution of the contents of the sample cell, was subtracted from the enthalpies of both the first and the second injections, to give the enthalpies of interaction of $\mathrm{Mg}^{2+}$ ions with the thylakoid membrane of the first and the second injections. The values for the first and second injections were summed and divided by the amount of chlorophyll in the sample $(1.44 \mathrm{~mL} \times 0.2 \mathrm{mM}=288 \mathrm{nmol})$, to give the molar enthalpy of the total interaction of $\mathrm{Mg}^{2+}$ ions with the thylakoid membrane. The values for all experiments with no added trypsin performed on a particular day were averaged, as were those obtained on that same day with added trypsin. The average molar enthalpy of experiments performed with trypsin (shown by subsequent fluorescence analysis not to have stacked) was then subtracted from that of experiments performed without trypsin 

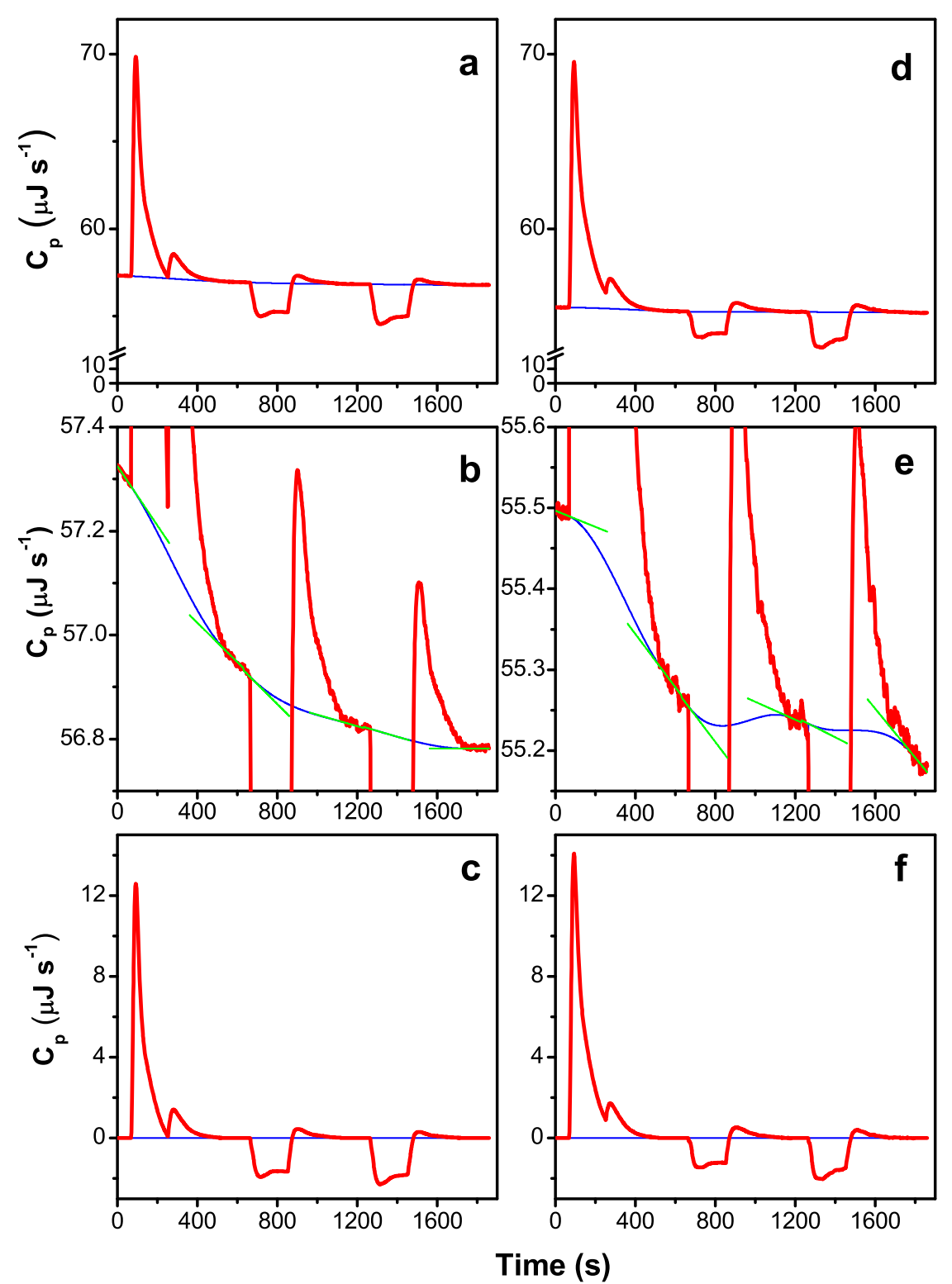

Figure 5 Heat-capacity $\left(\mathrm{C}_{\mathrm{p}}\right)$ plots and extrapolated baselines obtained from injection of $\mathrm{MgCl}_{2}$ into thylakoid suspensions which did not contain trypsin or which did contain trypsin. [Chl] in the suspensions was $0.2 \mathrm{mM}$. $\left[\mathrm{MgCl}_{2}\right]$ in the syringe was $25 \mathrm{mM}$. Three injections, each of $95 \mu \mathrm{Ls}$ and duration $190 \mathrm{~s}$, were made at $60 \mathrm{~s}, 660 \mathrm{~s}$ and 1,260 s. Heat capacity plots obtained from thylakoid suspensions which (a) did not contain trypsin or (d) contained trypsin at a 1:3,000 molar ratio of trypsin to chlorophyll. (b) and (e) are expanded segments of the same plots shown in (a) and (d). The thick red curves denote the raw ITC data and the thin blue curves denote the extrapolated baselines, calculated as described in Methods. The green straight lines in (b) and (e) denote the linear fits made to the segments of the plot immediately before each injection and at the end of each injection. (c) and (f) are the plots shown in (a) and (d) after the baselines have been subtracted. The thick red curves denote the data plots and the thin blue lines denote the $\mathrm{x}$-axes.

(shown by subsequent fluorescence analysis to have stacked) to give the average enthalpy change of membrane stacking for that day. The enthalpy changes of membrane stacking for six days were averaged (Column 1, Table 1). The large relative error is due to the enthalpy change being actually the near-zero difference of two large enthalpies which must be subtracted one from the other, resulting in an absolute error which is large in comparison.

Calculation of the theoretical component enthalpy changes of membrane stacking. The theoretical calculations made by Sculley et al. $(1980)^{18}$ and Rubin et al. $(1981)^{21}$ of the various forces between thylakoid membranes as functions of intermembrane distance are reproduced on a linear scale in Figure 2. The theoretical enthalpy changes of stacking produced by these forces, represented by the areas under the curves, were calculated by integration of these curves, yielding values in units of $J$ $(\mathrm{nm})^{-2}$ of membrane area. Each Chl molecule corresponds to a thylakoid membrane area of $1.71 \pm 0.14 \mathrm{~nm}^{2}$ for spinach ${ }^{31}$. However, the units of membrane area in Figure 2 are really units of area for a pair of stacked membranes and therefore represent double units of membrane surface area. Thus, $1.71 \mathrm{~nm}^{2}$ of the double membrane of Figure 3 really corresponds to 2 chlorophyll molecules. The enthalpy changes obtained by integrating the curves in Figure 2 were therefore multiplied by $0.5 \times 1.71 \mathrm{~nm}^{2}$ molecule ${ }^{-1} \mathrm{Chl}$ and then multiplied by Avogadro's number to give units of $\mathrm{J} \mathrm{mol}^{-1} \mathrm{Chl}$.

Approximate estimates of the electrostatic enthalpies at membrane separation (Gap) distances lower than for which the forces were calculated by Rubin $e t$ al.

$(1981)^{21}$ were obtained by extrapolation. The slopes of the semi-log plots shown in Figure 8 of Rubin et al. ${ }^{21}$ were measured at the lowest Gap distances for which they had been calculated: $3.3 \mathrm{~nm}$ for the electrostatic forces. The semi-log plots were then, using straight lines, extrapolated back to $2 \mathrm{~nm}$. Enthalpies were obtained by integration.

Calculation of the electric field strength near negatively-charged areas of the stromal surface of LHC-II trimers. For a negatively-charged surface (surface charge density of magnitude $|\sigma|)$ bathing in a mixed electrolyte of valence types $\left(\mathrm{Z}^{+} / \mathrm{Z}^{-} ; \mathrm{Z}^{2+}\right.$ I $\left.\mathrm{Z}^{-}\right)$, such as ( $\mathrm{KCl}$, bulk monovalent cation concentration $c_{1, \infty} ; \mathrm{MgCl}_{2}$, bulk divalent cation concentration $c_{2, \infty}$ ), the Gouy-Chapman theory (Equation A17 in Rubin and 
Barber, $1980^{17}$ ) gives the magnitude of the electric field strength $E$ as a function of distance $x$ from the surface:

$$
E=\sqrt{\left(2 R T \gamma^{2}\right) /\left(\varepsilon_{0} \varepsilon_{r}\right)}\left(\frac{v}{v^{2}-c_{2, \infty}}\right) \operatorname{sech}^{2}\left(\phi_{0}+\kappa x\right)
$$

In this equation $\gamma=c_{1, \infty}+3 c_{2, \infty}$

$\kappa=\sqrt{\left(F^{2} \gamma\right) /\left(2 R T \varepsilon_{0} \varepsilon_{r}\right)}$ where $F$ is the Faraday constant, $R$ the gas constant, $T$ the absolute temperature, $\varepsilon_{0}$ the permittivity of a vacuum, and $\varepsilon_{r}$ the dielectric constant. $\phi_{0}=\tanh ^{-1}\left(v_{0} / \sqrt{\gamma}\right)$ where $v_{0}=\sqrt{c_{2, \infty}+\left(c_{1, \infty}+2 c_{2, \infty}\right) e^{-\left(F \psi_{0}\right) /(R T)}}, \psi_{0}$ being the surface electric potential (Equation 8 of Rubin and Barber, $1980^{17}$ ). For a $\mathrm{Z}^{+} / \mathrm{Z}^{-}$ electrolyte at a bulk concentration $20 \mathrm{mM}\left(=20 \mathrm{~mol} \mathrm{~m}^{-3}\right.$, unstacking medium $), \psi_{0}$ is given by

$$
|\sigma|=\sqrt{2 R T \varepsilon_{0} \varepsilon_{r}\left(20 e^{-F \psi_{0} /(R T)}+20 e^{F \psi_{0} /(R T)}-2\right)}
$$

For a $\mathrm{Z}^{+} / \mathrm{Z}^{-} ; \mathrm{Z}^{2+} / \mathrm{Z}^{-}$electrolyte $(10 \mathrm{mM} \mathrm{KCl}+10 \mathrm{mM} \mathrm{NaOH}$ added to adjust the $\mathrm{pH}$ of the $20 \mathrm{mM}$ Hepes buffer; $5 \mathrm{mM} \mathrm{MgCl}$, stacking medium), $\psi_{0}$ is given by:

$$
|\sigma|=\sqrt{2 R T \varepsilon_{0} \varepsilon_{r}\left[\left(20 e^{-F \psi_{0} /(R T)}-1\right)+\left(30 e^{F \psi_{0} /(R T)}-1\right)+\left(5 e^{-2 F \psi_{0} /(R T)}-1\right)\right]}
$$

An estimate of the net $\sigma$ for the whole thylakoid membrane is $-0.025 \mathrm{C} \mathrm{m}^{-2}$, resulting from a mix of negative and positive surface charges ${ }^{5,32}$. Assuming that the local $|\sigma|$ of a negatively-charged area on the stroma-facing surface of LHC-II is ten times the net $\sigma$, the local $\psi_{0}$ is the value that results in the right-hand expression in Equation (2) or (3) being equal to $|\sigma|$.

$$
\text { Finally, } v=\sqrt{\gamma} \tanh \left(\phi_{0}+\kappa x\right)
$$

1. Anderson, J. M. The significance of grana stacking in chlorophyll $b$-containing chloroplasts. Photobiochem. Photobiophys. 3, 225-241 (1982).

2. Trissl, H.-W. \& Wilhem, C. Why do thylakoid membranes from higher plants form grana stacks? Trends Biochem. Sci. 18, 415-419 (1993).

3. Anderson, J. M. Insights into the consequences of grana stacking of thylakoid membranes in vascular plants: a personal perspective. Aust. J. Plant Physiol. 26, 625-639 (1999).

4. Chow, W. S., Kim, E.-H., Horton, P. \& Anderson, J. M. Granal stacking of thylakoid membranes in higher plant chloroplasts: the physiochemical forces at work and the functional consequences that ensue. Photochem. Photobiol. Sci. 4, 1081-1090 (2005).

5. Barber, J. Influence of surface charges on thylakoid structure and function. Ann. Rev. Plant Physiol. 33, 261-95 (1982).

6. Andersson, B. \& Anderson, J. M. Lateral heterogeneity in the distribution of chlorophyll-protein complexes of the thylakoid membranes of spinach chloroplasts. Biochim. Biophys. Acta 593, 427-440 (1980).

7. Anderson, J. M. Consequences of spatial separation of Photosystem 1 and 2 in thylakoid membranes of higher plant chloroplasts. FEBS Lett. 124, 1-10 (1981).

8. Chow, W. S. in Advances in Photosynthesis Research Sybesma C. (ed.) Vol. III 83-86 (Dr W. Junk Publishers, The Hague, 1984).

9. Horton, P. Are grana necessary for regulation of light harvesting? Aust. J. Plant Physiol. 26, 659-669 (1999).

10. Anderson, J. M. \& Aro, E.-M. Grana stacking and the protection of photosystem II membranes of higher plant leaves under sustained high irradiance: an hypothesis. Photosynth. Res. 41, 315-326 (2004).

11. Joliot, P. \& Joliot, A. Cyclic electron transfer in plant leaf. Proc. Natl. Acad. Sci. USA 99, 10209-10214 (2002).

12. Israelachvili, J. N. \& Adams, G. E. Direct measurement of long-range forces between two mica surfaces in aqueous $\mathrm{KNO}_{3}$ solutions. Nature 262, 774-776 (1976).

13. Daum, B., Nicastro, D., Austin, J. II., McIntosh, J. R. \& Kühlbrandt, W. Arrangement of photosystem II and ATP synthase in chloroplast membranes of spinach and pea. Plant Cell 22, 1299-1312 (2010).

14. Chow, W. S. Grana formation: entropy-assisted local order in chloroplasts? Aust. J. Plant Physiol. 26, 641-647 (1999).

15. Kim, E.-H., Chow, W. S., Horton, P. \& Anderson, J. M. Entropy-assisted stacking of thylakoid membranes. Biochim. Biophys. Acta 1708, 187-195 (2005).

16. Duniec, J. T., Sculley, M. J. \& Thorne, S. W. An analysis of the effect of mono- and di-valent cations on the forces between charged lipid membranes with special references to the grana thylakoids of thylakoids. J. Theor. Biol. 79, 473-484 (1979).
17. Rubin, B. T. \& Barber, J. The role of membrane surface charge in the control of photosynthetic processes and the involvement of electrostatic screening. Biochim. Biophys. Acta 592, 87-102 (1980).

18. Sculley, M. J., Duniec, J. T., Thorne, S. W., Chow, W. S. \& Boardman, N. K. The stacking of chloroplast thylakoids. Quantitative analysis of the balance of forces between thylakoid membranes of chloroplasts, and the role of divalent cations. Arch. Biochem. Biophys. 201, 339-346 (1980).

19. Izawa, S. \& Good, N. E. Effect of salts and electron transport on the conformation of isolated chloroplasts. II. Electron microscopy. Plant Physiol. 41, 544-552 (1966).

20. Gross, E. L. \& Prasher, S. H. Correlation between monovalent cation induced decrease in chlorophyll $a$ fluorescence and chloroplast structural changes. Arch. Biochem. Biophys. 164, 460-468 (1974).

21. Rubin, B. T., Chow, W. S. \& Barber, J. Experimental and theoretical considerations of mechanisms controlling cation effects on thylakoid membrane stacking and chlorophyll fluorescence. Biochim. Biophys. Acta 634, 174-190 (1981).

22. Mullet, J. E., Baldwin, T. O. \& Arntzen, C. J. A mechanism for chloroplast thylakoid adhesion mediated by the $\mathrm{Chl} a / b$ light harvesting complex. In Akoyunoglou, G. (ed.) Photosynthesis III. Structure and Molecular Organisation of the Photosynthetic Apparatus (Balaban International Science Services, Philadelphia) 577-582 (1981)

23. Jennings, R. C., Forti, G., Gerola, P. D. \& Garlaschi, F. M. Studies on cationinduced thylakoid membrane stacking, fluorescence yield, and photochemical efficiency. Plant Physiol. 62, 879-884 (1978).

24. Standfuss, J., Terwisscha van Scheltinga, A. C., Lamborghini, M. \& Kühlbrandt, W. Mechanisms of photoprotection and nonphotochemical quenching in pea light-harvesting complex at $2.5 \AA$ resolution. EMBO J. 24, 919-928 (2005).

25. Nelson, P. Biological Physics. Energy, Information and Life 265-272 (W. H. Freeman \& Co., New York, USA, 2004).

26. Dinsmore, A. D., Yodh, A. G. \& Pine, D. J. Entropic control of particle motion using passive surface structures. Nature 383, 239-242 (1996).

27. Hugly, S., Kunst, L., Browse, J. \& Somerville, C. Enhanced thermal tolerance of photosynthesis and altered chloroplast structure in a mutant of Arabidopsis deficient in lipid desaturation. Plant Physiol. 90, 1134-1142 (1989).

28. Bhaya, D. \& Jagendorf, A. T. Optimal conditions for translation by thylakoidbound polysomes from pea chloroplasts. Plant Physiol. 75, 832-838 (1984).

29. Chow, W. S., Thorne, S. W., Duniec, J. T., Sculley, M. J. \& Boardman, N. K. The stacking of chloroplast thyakoids. Effects of cation screening and binding, studied by the digitonin method. Arch. Biochem. Biophys. 201, 347-355 (1980).

30. Porra, R. J., Thompson, W. A. \& Kriedemann, P. E. Determination of accurate extinction coefficients and simultaneous equations for assaying chlorophylls $a$ and $b$ extracted with four different solvents: verification of the concentration of chlorophyll standards by atomic absorption spectroscopy. Biochim. Biophys. Acta 975, 384-394 (1989).

31. Kirchhoff, H., Mukherjee, U. \& Galla, H.-J. Molecular architecture of the thylakoid membrane: Lipid diffusion space for plastoquinone. Biochem. 41, 4872-4882 (2002).

32. Chow, W. S., Miller, C. \& Anderson, J. M. Surface charges, the heterogeneous distribution of the two photosystems, and thylakoid stacking. Biochim. Biophys. Acta 1057, 69-77 (1991).

\section{Acknowledgments}

We thank Prof. Jan M. Anderson FRS for helpful comments on the manuscript. This work was supported consecutively by Australian Research Council grants (DP0664719 and DP 1093927)

\section{Author contributions}

H.J., J.R.L. and W.S.C. designed research; H.J. and J.R.L. performed research; H.J., J.R.L. and W.S.C. analyzed data; and J.R.L. and W.S.C. wrote the paper.

\section{Additional information}

Competing financial interests: The authors declare no competing financial interests.

How to cite this article: Jia, H., Liggins, J.R. \& Chow, W.S. Entropy and biological systems: Experimentally-investigated entropy-driven stacking of plant photosynthetic membranes. Sci. Rep. 4, 4142; DOI:10.1038/srep04142 (2014).

This work is licensed under a Creative Commons AttributionNonCommercial-ShareAlike 3.0 Unported license. To view a copy of this license, visit http://creativecommons.org/licenses/by-nc-sa/3.0 